\title{
Controllable Synthesis and Electroluminescence Property of Water-Sol ZnCdTe Alloy for Light-emitting Diodes
}

\author{
Yan Wang, Yanbing Hou, Haixin Cui, Changjiao Sun, and Yunzhang Lu
}

\begin{abstract}
By changing the stabilizer system and synthesis temperature, $\mathrm{ZnCdTe}$ alloy have been controllably synthesized in aqueous solution using a simple route. A high synthesis temperature leads to nanoparticles growth using thioglycolic acid (TGA) as the stabilizer, whereas a low synthesis temperature promotes the form of nanorods using TGA and L-Cysteine( L-Cys )as the stabilizer. The different morphologies of samples have been characterized by transmission electron microscopy (TEM). The optical properties of the obtained ZnCdTe nanorods and nanoparticles have been investigated by photoluminescence (PL) spectroscopy with different refluxing times. We have also demonstrated electroluminescence (EL) property from heterostructure device fabricated with the prepolymer of Poly(p-phenylenevinylene) (PPV ) and ZnCdTe alloyed nanocrystals. Current density of device with $\mathrm{ZnCdTe}$ alloy in emitting layer is obviously increased.
\end{abstract}

Index Terms-Optical properties, electroluminescence, ZnCdTe, alloy.

\section{INTRODUCTION}

Nanostructured materials, especially one-dimensional (1D) materials such as nanowires, nanorods and nanotubes have been the focus of intensive research[1], [2]. The ability to rationally tune the shape of colloidal nanocrystals from spheres to rods/wires has been actively sought during the last several years. Up to now, many methods have been developed to achieve 1D growth, including metal particle-initiated 1D uniaxial growth [3], [4], and template-directed 1D growth [5], [6]. Besides these important methods, exploring new and simple wet synthetic routes without using metal seeds and templates for achieving 1D growth of semiconductor nanomaterials is also one of the focuses of intensive research.

Among various II - VI semiconductor materials, CdTe have attracted much attention of the scientists because such material has shown significant potentials to be used in biological labeling [7], light-emitting devices[8], and solar cells[9]. Size-tunable properties of CdTe nanocrystals have been investigated by many world-wide research groups [10], [11]. However, the tuning of physical and chemical properties of binary nanocrystals is limited by only changing the particle size, which causes problems in many applications such as biological labeling, superlattice structures, and

Manuscript received November 10, 2011; revised November 20, 2011.

Yan Wang, Haixin Cui, Changjiao Sun are with Institute of Environment and Sustainable Development in Agriculture, Chinese Academy of Agricultural Sciences, No.12 South Street of Zhongguancun Haidian District in Beijing, China.

Yanbing Hou and Yunzhang $\mathrm{Lu}$ are with Key Laboratory of Luminescence and Optical Information, Ministry of Education, Institute of Optoelectronic Technology, Beijing Jiaotong University, Beijing, China. nanoelectronics[12], [13]. This is because very small nanocrystals is hard to passivate and exhibit unstable optical properties. Recent advances in ternary composition-tunable semiconductor nanocrystals have attracted attention of scientists[12]-[16]. As has been clarified in our previous studies[17], ternary $\mathrm{Zn}_{\mathrm{x}} \mathrm{Cd}_{1-\mathrm{x}} \mathrm{Te}$ nanocrystals can be synthesized successfully. Currently, some interesting results have been obtained in further research, and we found the shape of ZnCdTe nanocrystals can be controlled by changing the stabilizer system and synthesis temperature. Ternary $\mathrm{ZnCdTe}$ nanorods and nanoparticles can be shape-controllable synthesized in aqueous media by a simple route. Our method is of particular interest due to the controllability of compositions, low temperature, simple facilities, low cost, good homogenization, better water-soluble, shape-controllable and bio-compatible. Furthermore, we investigated the electroluminescence property of hererostructure devices fabricated with $\mathrm{ZnCdTe}$ alloy and prepolymer PPV, which were used as an emitting layer.

\section{SyNTHESIS OF ZNCDTE NANOPARTICLES AND NANORODS}

Mixed precursor solutions were prepared firstly by mixing $\mathrm{Cd}\left(\mathrm{CH}_{3} \mathrm{COO}\right)_{2} \cdot 2 \mathrm{H}_{2} \mathrm{O}$ and $\mathrm{Zn}\left(\mathrm{CH}_{3} \mathrm{COO}\right)_{2} \cdot 2 \mathrm{H}_{2} \mathrm{O}$ solution. Then the stabilizers were added to the mixed precursor solutions under $\mathrm{N}_{2}$ flow. The precursor solutions were adjusted to $\mathrm{pH} 9$ by $\mathrm{NaOH}$ solution. Then, oxygen-free NaHTe solution, which was freshly prepared from tellurium powder and $\mathrm{NaBH}_{4}$ in water, was injected into the above solution under vigorous stirring. The resulting mixture solution was refluxed in fixed temperature for different times. We found $\mathrm{ZnCdTe}$ nanoparticles were produced with using TGA as the stabilizer when the refluxed temperature is above $80^{\circ} \mathrm{C}$, and $\mathrm{ZnCdTe}$ nanorods were produced with using TGA and L-Cys mixed stabilizer system in lower temperature. It is a simple route to achieve shape-controlled synthesis of $\mathrm{ZnCdTe}$ nanoparticles and nanorods.

\section{DEVICE FABRICATION}

ITO-coated glass substrate was first cleaned for sample preparation by detergent and distilled water in an ultrasonic bath, respectively. A hole injection layer of poly(3,4-ethylenedioxythiophene): poly(styrenesulfonate) (PEDOT:PSS) was spin-coated onto a cleaned ITO glass substrates and the layer then was heated to $80^{\circ} \mathrm{C}$ for about $10 \mathrm{~min}$. The PEDOT:PSS layer was used to improve the holes transport. The $\mathrm{ZnCdTe}$ alloyed nanocrystals and prepolymer PPV were dissolved in distilled water, and then the blend was 
spin-coated onto the top of PEDOT:PSS laye with a thickness of about 50nm. The organic films of BCP $(11 \mathrm{~nm})$ and Alq3 $(9 \mathrm{~nm})$ was vapor-deposited onto the nanocomposite layer as the hole-blocking layer and the electron-transporting layer by the thermal evaporation method, respectively.

\section{Characterization}

Transmission electron microscopy (TEM) images were performed on a HITACHI H-700 transmission electron microscope. X-ray diffraction (XRD) pattern was measured by Philips APD-10 X-ray diffractometer with graphite monochromatized $\mathrm{Cu} \mathrm{Ka}$ radiation $(\lambda=0.154178 \mathrm{~nm})$. PL spectra were performed by a SPEX Flurolog-3 Spectrofluorimeter. All the measurements were performed at room temperature under ambient atmosphere. Cyclic voltammetry (CV) measurement was performed on a CHI660A electrochemical workstation. Current-voltage curves of the devices were measured using a Keithley 2410 source meter.

\section{RESUlts AND DisCUSSION}

TEM images of TGA-stabilized ZnCdTe nanoparticles reacted for 30min were shown in Fig.1, TEM image shows clearly that the morphology of $\mathrm{ZnCdTe}$ nanoparticles using TGA as the stabilizer is close to spherical. As shown in Fig. 1, the $\mathrm{ZnCdTe}$ nanoparticles is small obtained for refluxing $30 \mathrm{~min}$ and the average particle size is about $3.8 \mathrm{~nm}$ in diameter. The average particle size of samples increases to about $5.4 \mathrm{~nm}$ with continually increasing time from $30 \mathrm{~min}$ to $120 \mathrm{~min}$. There is not obvious size difference for $\mathrm{ZnCdTe}$ nanoparticles reacted for more than $120 \mathrm{~min}$, which may due to dominant growth of $\mathrm{ZnCdTe}$ nanoparticles occurred in the early stages.

TEM images of ZnCdTe nanorods stabilized by TGA and L-Cys mixed stabilizers in the lower growth temperature with different refluxing times (30min and 60min) were shown in Fig.2a-b. We can observe the formation of $\mathrm{ZnCdTe}$ nanorods under this experimental condition. The diameters of the short and the long axis of the samples remained at about 20-40 and 30-100 nm, respectively (in Fig.2).

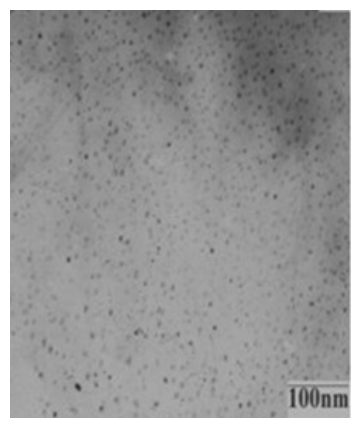

Fig. 1. TEM images of $\mathrm{ZnCdTe}$ nanoparticles stabilized by TGA reacted for $30 \mathrm{~min}$.

In the TGA and L-Cys mixed system, partial removal of stabilizer (TGA) can decrease the mutual electrostatic repulsion of the products. As a result, the dipole attraction is favorable for $1 \mathrm{D}$ growth with $-\mathrm{NH}_{2}$ and $-\mathrm{COO}_{2}$ of L-Cys acting as a pair of binding sites for metallic ions[18]. Once the small $\mathrm{ZnCdTe}$ nanocrystals formed in the reaction system, the nanocrystals have a chance of combining with each other through the chelate function between the stabilizers and metallic ions. TGA and L-Cys mixed system made the growth orientation more restricted, suppressing other growth directions[2]. In addition, if OR which is a diffusion-limited process is suppressed properly, it should favor anisotropic growth of nanocrystals. In the aqueous process of growth of $\mathrm{ZnCdTe}$ nanorods, the nucleation occurs at low temperature. OR process may be suppressed at lower growth temperature, which leads to the formation of $\mathrm{ZnCdTe}$ nanorods[11]. As shown in Fig.2, the average size of $\mathrm{ZnCdTe}$ nanorods becomes bigger with increasing the refluxing time from $30 \mathrm{~min}$ to $60 \mathrm{~min}$, which dues to the continuous growth of $\mathrm{ZnCdTe}$ nanorods with rasied time.

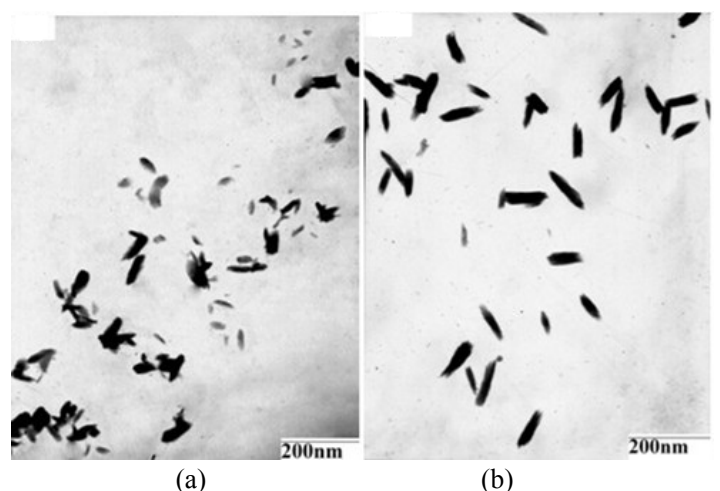

Fig. 2. TEM images of ZnCdTe nanorods stabilized by TGA and L-Cys with different refluxing times (a) $30 \mathrm{~min}$ (b) $60 \mathrm{~min}$.

The crystallographic properties of the as-synthesized ZnCdTe nanocrystals were determined using powder X-ray diffraction (XRD). As an example, the XRD pattern of the as-synthesized ZnCdTe nanocrystals was shown in Fig.3. The crystal structures can be assigned as cubic structures with the presence of characteristic (llll 111$),\left(\begin{array}{lll}2 & 2 & 0\end{array}\right)$, and (3 111$)$ peaks in the XRD patterns. XRD spectra show obvious broadening phenomena, which indicates the small size of the alloyed nanocrystals.

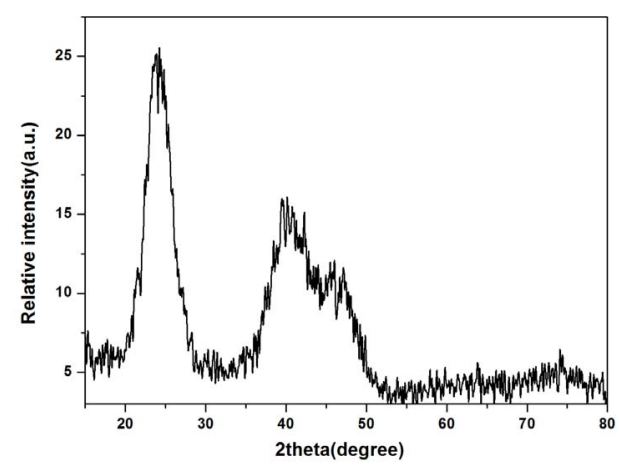

Fig. 3. XRD spectra of as-synthesized ZnCdTe nanocrystals.

As shown in Fig. 4, the photoluminescence (PL) spectra of $\mathrm{ZnCdTe}$ nanoparticles and nanorods were investigated with different refluxed times (30min, 60min, 120min). All obtained $\mathrm{ZnCdTe}$ nanoparticles and nanorods show strong luminescence. The PL peaks of $\mathrm{ZnCdTe}$ nanoparticles appeared at 513, 520 and $522 \mathrm{~nm}$, respectively. The full 
width half maxima (FWHM) of $\mathrm{ZnCdTe}$ nanoparticles is 40-44nm, indicating a relative narrow size distribution (Fig.4a). As mentioned above, the nanoparticles growth was accompanied by a red shift in the luminescence peaks. Fig. $4 \mathrm{~b}$ shows the PL spectra of $\mathrm{ZnCdTe}$ nanorods stabilized by L-Cys and TGA with different refluxing times. The full width half maxima (FWHM) of ZnCdTe nanorods is $50-58 \mathrm{~nm}$. Nanorod sample has green PL (peak: $540 \mathrm{~nm}$ ) with refluxing for $30 \mathrm{~min}$. With increasing refluxing time, $\mathrm{ZnCdTe}$ nanorods show the longer wavelength emission (peak: $560 \mathrm{~nm}$ ) with larger size due to quantum confinement effect. The red shift of nanorods with increased refluxing time may due to the elongation of two axises and the decrease of confinement in two dimensions [2].

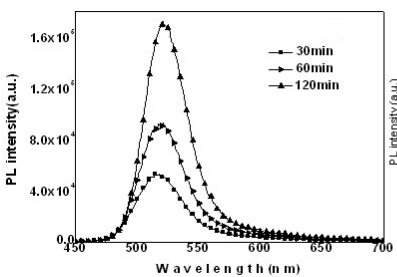

(a) (b)

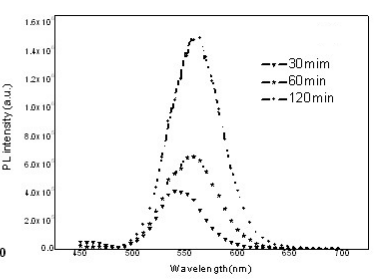

Fig. 4. (a)Photoluminescence spectra of TGA-stabilized ZnCdTe nanoparticles; (b)Photoluminescence spectra of TGA and L-Cys stabilized $\mathrm{ZnCdTe}$ nanorods.

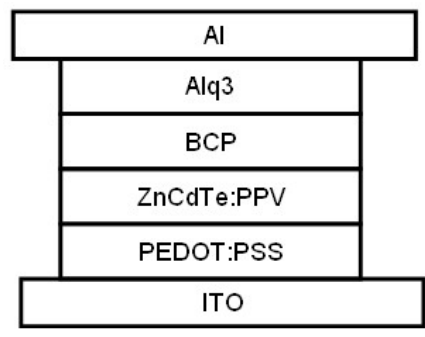

Fig. 5. Device configuration of the device.

Next we fabricated the heterostructure device by adding $\mathrm{ZnCdTe}$ alloyed nanocrystals into the prepolymer of Poly(p-phenylenevinylene) (PPV) as emitting layer. The schematic diagram of the device is shown in Fig. 5 In order to clarify the energy level of PPV prepolymer, a typical CV curve of PPV prepolymer solid film is first measured. As shown in Fig. 6, the oxidation peak and reduction peak were observed with good reproducibility, which corresponds to HOMO level of $5.44 \mathrm{eV}$ and LUMO level of $3.44 \mathrm{eV}$ calculated by formulas, respectively. The energy values were referenced against ferrocene as an internal standard where acetonitrile was used as a solvent [19], [20].

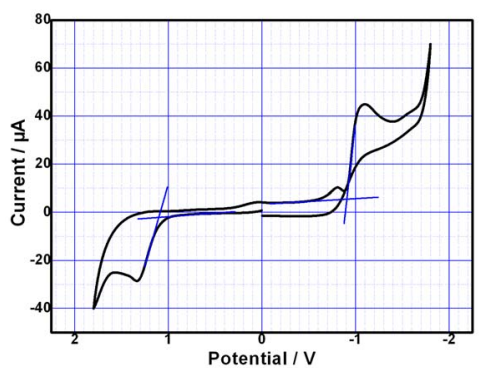

Fig. 6. Cyclic voltammogramm of a PPV prepolymer film on top of the Pt work electrode in a $0.1 \mathrm{M}$ solution of tetrabutylammonium perchlorate in acetonitrile.

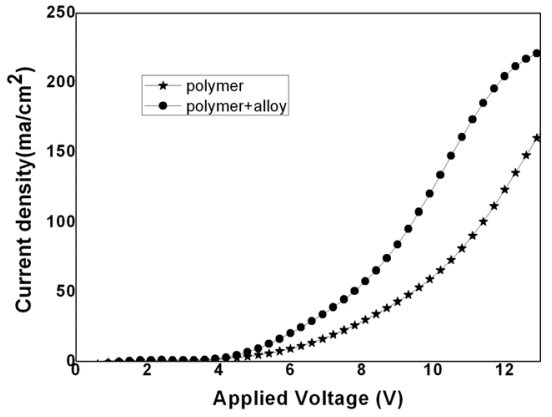

Fig. 7. Current versus voltage characteristic for devices with and without $\mathrm{ZnCdTe}$ alloy as the emitting layer.

Fig.7 shows the performance of the devices with and without $\mathrm{ZnCdTe}$ alloy as the emitting layer. As the result shown, current density of device with adding $\mathrm{ZnCdTe}$ is obviously increased comparing with that without adding $\mathrm{ZnCdTe}$ alloy. For PPV prepolymer, the carrier mobility is lower, which could result in lower electroluminescence efficiency of device. It favors to improve injection and transport of carrier and decrease the loss of combination process with a certain amount of $\mathrm{ZnCdTe}$ alloy in the nanocomposite, resulting in the increase of current density of device.

\section{CONCLUSION}

In conclusion, we have successfully synthesized $\mathrm{ZnCdTe}$ nanorods and nanoparticles in aqueous solution with changing the stabilizers and synthesis temperature using a facile route. The measurements of PL show the obtained samples have relative narrow photoluminescence FWHM and display few electronic defect PL properties. This new method for synthesizing $\mathrm{ZnCdTe}$ nanoparticles and nanorods in aqueous solutions is simple and environmentally friendly compared to organometallic approaches. We investigated the heterostructure device using the prepolymer PPV and $\mathrm{ZnCdTe}$ alloy as the emitting layer. The results showed that $\mathrm{ZnCdTe}$ alloy could improve the current intensity of the device.

\section{ACKNOWLEDGMENT}

We thank the Basic Scientific Research Fund of National Nonprofit Institutes (BSRF 201108) and Beijing Municipal Science \& Technology Commission (Nos. 4102046) for the financial support of this work. We thank Beijing Jiaotong University for help with optical property analysis.

\section{REFERENCES}

[1] Z. L. Wang, "Characterizing the Structure and Properties of Individual Wire-Like Nanoentities," Adv. Mater, vol. 12, no.17, pp.1295-1298, Sep. 2000

[2] J. Li, X. Hong, D. Li, K. Zhao, L. Wang, H. Z. Wang, Z .L. Du, J. H.Li, Y. B. Bai, and T .J. Li, "Mixed Ligands of Cysteine and Thioglycolic Acid Assisted Synthesis of Highly Luminescent Water-soluble CdTe Nanorods," Chem.Commun, vol. 15, pp.1740-1741, 2004.

[3] L. J. LauhonL, M. S. Gudiksen, D. L. Wang, and C. M. Lieber, "Epitaxial core-shell and core-multishell nanowire heterostructures," Nature, vol. 420, pp.57-61, 2002.

[4] S. Kan, T. Mokari, E. Rothenberge, and U. Banin, "Synthesis and size-dependent properties of zinc-blende semiconductor quantum rods," Nat. Mater, vol.2, pp.155-158, 2003.

[5] D. J. Peña, J. N.Mbindyo, A. J.Carado, T. E.Mallouk, C. D. Keating, B. Razavi, and T. S. J Mayer, "Template Growth of Photoconductive 
Metal-CdSe-Metal Nanowires. Phys," Chem. B, vol.106, pp. 7458-7462, 2002.

[6] D. W. Deng, Y. B. Qin, X. Yang, J. S. Yu, and Y. Pan, "The selective synthesis of water-soluble highly luminescent CdTe nanoparticles and nanorods: The influence of the precursor $\mathrm{Cd} / \mathrm{Te}$ molar ratio," Journal of Crystal Growth, vol. 296, pp. 141-149, 2006.

[7] J. Lee, A. O. Govorov, J. Dulka, and N. A. Kotov, “ Bioconjugates of CdTe nanowires and Au nanoparticles: Plasmon-exciton interactions, luminescence enhancement,and collective effects," Nano Lett, vol.4, pp. 2323-2330, 2004.

[8] N. P.Gaponik, D. V.Talapin, A. L. Rogach, and A. Eychmüller, "Electrochemical synthesis of CdTe nanocrystal/polypyrrole composites for optoelectronic applications," J. Mater. Chem, vol.10, pp.2163-2166, 2000.

[9] A. Guptaa, V. Parikha, and A. D. Compaan, "High efficiency ultra-thin sputtered CdTe solar cells," Solar Energy Materials \& Solar Cells, vol.90, pp.2263-2271, 2006.

[10] J. F. Weng, X. T. Song, L. Li, H. F. Qian, K.Y. Chen, X. M. Xu, C. X. $\mathrm{CAO}$, and J. C. Ren, "Highly luminescent CdTe quantum dots prepared in aqueous phase as an alternative fluorescent probe for cell imaging," Talanta, vol.70, pp.397-402, 2006.

[11] H. Zhang, D. Y. Wang, B. Yang, and MöHWALD, "Manipulation of Aqueous Growth of CdTe Nanocrystals To Fabricate Colloidally Stable One-Dimensional Nanostructures," J. AM. CHEM. SOC, vol. 128, pp.10171-10180, 2006

[12] X. H. Zhong, Z. H. Zhang, S. H. Liu, M. Y. Han, and W. Knoll, "Embryonic Nuclei-Induced Alloying Process for the Reproducible Synthesis of Blue-Emitting $\mathrm{Zn}_{\mathrm{x}} \mathrm{Cd}_{1-\mathrm{x}} \mathrm{Se}$ Nanocrystals with Long-Time Thermal Stability in Size Distribution and Emission Wavelength," $J$. Phys. Chem. B. vol. 108, pp.15552-15559, 2004.

[13] X. H. Zhong, M. Y. Han, and W. Knoll, "Composition-tunable $\mathrm{Zn}_{\mathrm{x}} \mathrm{Cd}_{1-\mathrm{X}} \mathrm{Se}$ nanocrystals with high luminescence and stability," J.Am.Chem. Soc., vol.125, pp. 8589-8594, 2003.

[14] B. Tang, F. Yang, Y. Lin, L. H. Zhuo, J. C. Ge, and L. H. Cao, "Synthesis and Characterization of Wavelength-Tunable, Water-Soluble, and Near-Infrared-Emitting CdHgTe Nanorods," Chem. Mater, vol. 19, pp. 1212-1214, 2007.

[15] X. H. Zhong, Y. Y. Feng, W. Knoll, and M. Y. Han, "Alloyed $\mathrm{Zn}_{\mathrm{x}} \mathrm{Cd}_{1-\mathrm{x}} \mathrm{S}$ Nanocrystals with Highly Narrow Luminescence Spectral Width,” J. Am. Chem. Soc., vol.125, pp. 13559-13563, 2003.

[16] M. W. Degroot, H. Ro"SNER, and J. F. Corrigan, "Control of Metal Ion Composition in the Synthesis of Ternary II-II'-VI Nanoparticles Using a Mixed-Metal Cluster Precursor Approach," Chem. Eur. J., vol.12, pp. $1547-1554,2006$

[17] Y. Wang, Y. B. Hou, A. W. Tang, B. Feng, Y. Li, J. Liu, and F. Teng, "Synthesis and optical properties of composition-tunable and water-soluble $\mathrm{Zn}_{\mathrm{x}} \mathrm{Cd}_{1-\mathrm{x}} \mathrm{Te}$ alloyed nanocrystals," Journal of Crystal Growth. vol.308, pp. 19-25, 2007.

[18] R. B. Wllson, P. D. Meester, and D. J. Hodgson, "Structural characterization of bis(L-methionato)zinc(II), Zn(L-met)2," Inorg. Chem., vol.16, pp.1498-1502, 1977.

[19] J. Pommerehne, H. Vestweber, W. Guss, R.F. Mahrt, H. Ba" ssler, M. Porsch, and J. Daub, "Efficient two layer leds on a polymer blend basis," Adv. Mater., vol. 7,pp 551-554, 1995.

[20] J. Y. Sun, Z. Q. He, L. P. Mu, X. Han, J. J. Wang, B. Wang, C. J. Liang, Y. S. Wang, Y. L. Liu, and S. K. Cao, "Preliminary photovoltaic response from a polymer containing p-vinylenephenylene amine backbone," Solar Energy Materials \& Solar Cells, vol. 91, 1289-1298, 2007.

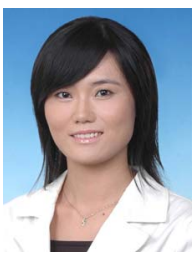

Yan Wang was born on June 24, 1982 in China. assistant professor, the main research is controllable synthesis of semiconductor nanocrystals and performance of photoelectronic device based on the blends of semiconductor nanocrystals and polymer. The educational background is listed as follows: 2010-present, she is an ASSISTANT PROFESSOR in Institute of Environment and Sustainable Development in Agriculture, Chinese Academy of Agricultural Sciences, Beijing, China. Research interest of her group is in the synthesis and functionalization of nanostructured materials and their applications in biotechnology, agriculture and photoelectronic device.

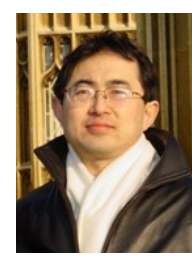

Yanbing Hou was born in China. 2003.8- , Institute of Optoelectronic Technology, Beijing Jiaotong University, Beijing, China. 2003.8-present, he is a Professor in Institute of Optoelectronic Technology, Beijing Jiaotong University. Research interest of his group is organic/inorganic thin-film light emitting diode (LED), thin-film electroluminescence, up-conversion luminescence of rare earth doped compound, polymer light emitting devices, electrooptical device, polymer photoamplifier and behavioral study of polymer excited state under electric fields.

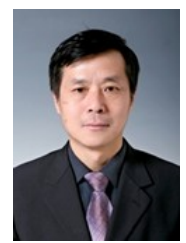

Haixin Cui was born on June, 1963 in China. Ph.D., Osaka Prefecture University; Research Associate and Ph.D. supervisor, Institute of Environment and Sustainable Development in Agriculture, Chinese Academy of Agricultural Sciences; Director of Nanotechnology research center of Agriculture, CAAS; Academic leader of Horticultural Facilities and Biological Physics, CAAS. Dr. Cui served as Professional Committee member of China Medicinal Biotechnology Association Nanobiotechnology branch, Professional Committee member of Chinese Society of Agricultural Engineering Horticultural Facilities branch, evaluation expert of The award of national science and Technology Conference, evaluation expert of "863" Program, evaluation expert of Chinese National Natural Science Foundation, budget evaluation expert of National Key Technology R\&D Program.

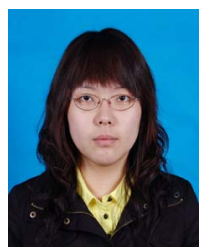

Changjiao Sun was born on November 16,1984 in China. Intern Researcher, the main research is application of nanotechnology in agriculture. The education background is listed as follow:M.S. 2007-2010, Biophysics, Chinese Academy of Agricultural Sciences, Beijing, China.B.E., 2003-2007, Biological Engineering, Beijing Technology and Business University, Beijing, China

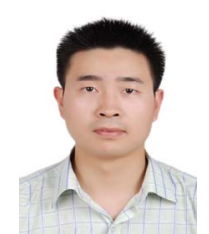

Yunzhang Lu was born on September 21, 1984 in China. PhD candidate, the main research is Organic Solar Cell. The education background is listed as follow: M.S., 2007-2009, Optical Engineering, Beijing Jiaotong University, Beijing, China. B.E., 2003-2007, Optical Information Science and Technology, Southwest University of Science and Technology, Sichuan, China. 2009-present, he is a $\mathrm{PhD}$ candidate in Institute of Optoelectronic Technology, Beijing Jiaotong University, Beijing, China. 